\title{
Exact travelling wave solutions of the symmetric regularized long wave (SRLW) using analytical methods
}

\author{
Jalil Manafian ${ }^{1, *}$ and Isa Zamanpour ${ }^{2}$ \\ ${ }^{1}$ Department of Mathematics, College of Mathematics, Ahar Branch, Islamic Azad University, \\ Ahar, Iran. \\ ${ }^{2}$ Department of Mathematics, College of Mathematics, Karaj Branch, Islamic Azad University, \\ Karaj, Iran.
}

Received 9 February 2014; Accepted 14 February 2014

Editor: David G. Yu

\begin{abstract}
In this article, we establish exact travelling wave solutions of the symmetric regularized long wave (SRLW) by using analytical methods. The analytical methods are: the tanh-coth method and the $\operatorname{sech}^{2}$ method which used to construct solitary wave solutions of nonlinear evolution equations. With the help of symbolic computation, we show that aforementioned methods provide a straightforward and powerful mathematical tool for solving nonlinear partial differential equations.
\end{abstract}

Keywords Symmetric regularized long wave (SRLW); The tanh-coth method; The sech ${ }^{2}$ method

DOI: $10.19139 /$ soic.v2i1.69

\section{Introduction}

Recently, the investigation of exact travelling wave solutions to nonlinear partial differential equations plays an important role in the study of nonlinear modelling physical phenomena. Also the study of the travelling wave solutions plays an important role in nonlinear science. Nonlinear evolution equations are widely used as models to describe complex physical phenomena and have a significant role in

\footnotetext{
${ }^{*}$ Correspondence to: Department of Mathematics, College of Mathematics, Ahar Branch, Islamic Azad University, Ahar, Iran. Email: j-manafian@iau-ahar.ac.ir, Tel:+989147738166.
}

ISSN 2310-5070 (online) ISSN 2311-004X (print)

Copyright (C) 2014 International Academic Press 
several scientific and engineering fields. A variety of powerful methods has been presented, such as Hirota's bilinear method [1], the inverse scattering transform [2], sine-cosine method [3], homotopy perturbation method [19], homotopy analysis method [5, 6], variational iteration method $[7,8,9]$, the $\left(\frac{G^{\prime}}{G}\right)$-expansion method [10, 11], tanh-function method [12], tanhcoth method [13], Bäcklund transformation [14, 15], Exp-function method [16, 17, 18, 19, 20] and so on. In this article, we used the analytical methods to investigate the symmetric regularized long wave (SRLW) [18] in the following form

$$
u_{t t}+u_{x x}+u u_{x t}+u_{x} u_{t}+u_{x x t t}=0,
$$

which arises in several physical applications including ion sound waves in plasma [18]. The article is organized as follows: In Sections 2 and 3 first we briefly give the steps of the methods and apply the methods to solve the nonlinear partial differential equations. In Section 4, the application of the analytical methods to the symmetric regularized long wave (SRLW) will be introduced briefly. Also a conclusion is given in Section 5. Finally some references are given at the end of this paper.

\section{Basic idea of tanh-coth method}

The standard tanh method is well-known analytical method which first presented by Malfliet's [21] and developed in [21, 22]. By summarizing tanh-coth method by Wazwaz [23] for a given NLPDE with independent variables $X=(x, y, z, t)$ and dependent variable $u$ :

$$
\mathcal{P}\left(u, u_{t}, u_{x}, u_{y}, u_{z}, u_{x x}, u_{y y}, u_{z z}, u_{x y}, u_{t t}, u_{t x}, u_{t y}, u_{t z} \ldots\right)=0,
$$

can be converted to on ODE

$$
\mathcal{Q}\left(u,-c u^{\prime}, u^{\prime}, u^{\prime}, u^{\prime}, u^{\prime \prime}, \ldots\right)=0,
$$

which transformation $\xi=k_{1} x+k_{2} y-c t$ is wave variable. Also, $c, k_{1}$ and $k_{2}$ are constants to be determined later. Introducing a new independent variable

$$
Y=\tanh (\mu \xi), \quad \xi=k_{1} x+k_{2} y-c t,
$$

leads to the change of derivatives

$$
\begin{aligned}
\frac{d}{d \xi} & =\mu\left(1-Y^{2}\right) \frac{d}{d Y} \\
\frac{d^{2}}{d \xi^{2}} & =\mu^{2}\left(1-Y^{2}\right)\left(-2 Y \frac{d}{d Y}+\left(1-Y^{2}\right) \frac{d^{2}}{d Y^{2}}\right),
\end{aligned}
$$




$$
\frac{d^{3}}{d \xi^{3}}=\mu^{3}\left(1-Y^{2}\right)\left(\left(6 Y^{2}-2 Y\right) \frac{d}{d Y}-6 Y\left(1-Y^{2}\right) \frac{d^{2}}{d Y^{2}}+\left(1-Y^{2}\right)^{2} \frac{d^{3}}{d Y^{3}}\right) .
$$

The tanh-coth method [24] admits the use of the finite expansion

$$
u(\mu \xi)=S(Y)=\sum_{k=0}^{m} a_{k} Y^{k}+\sum_{k=1}^{m} b_{k} Y^{-k}
$$

where $a_{k}(k=0,2, \ldots, m), b_{k}(k=1,2, \ldots, m)$ and $\mu$ are constants to be determined later, but the degree of which is generally equal to or less than $m-1$, the positive integer $m$ can be determined by considering the homogeneous balance between the highest order derivatives and nonlinear terms appearing in Eq. (3). If $m$ is not an integer, then a transformation formula should be used to overcome this difficulty. For aforementioned method, expansion (7) reduces to the standard tanh method [21] for $b_{k}=0,1 \leq k \leq m$. Substituting Eq. (7) into the ODE results is an algebraic system of equations in the powers of $Y$ that will lead to the determination of the parameters $a_{k}(k=0,2, \ldots, m), b_{k}(k=1,2, \ldots, m)$ and $c$. To show the efficiency of the method described in the previous part, we present some examples.

\section{Basic idea of $\operatorname{sech}^{2}$ method}

We now describe the $\operatorname{sech}^{2}$ method for the given partial differential equations. We give the detailed description of method which to use this method, we take following steps:

Step 1. For a given NLPDE with independent variables $X=(x, y, z, t)$ and dependent variable $u$, we consider a general form of nonlinear equation:

$$
\mathcal{P}\left(u, u_{t}, u_{x}, u_{y}, u_{z}, u_{x x}, u_{y y}, u_{z z}, u_{x y}, u_{t t}, u_{t x}, u_{t y}, u_{t z} \ldots\right)=0,
$$

which can be converted to on ODE

$$
\mathcal{Q}\left(u,-c u^{\prime}, u^{\prime}, u^{\prime}, u^{\prime}, u^{\prime \prime}, \ldots\right)=0
$$

which transformation $\xi=k_{1} x+k_{2} y-c t$ is wave variable. Also, $c, k_{1}$ and $k_{2}$ are constants to be determined later.

Step 2. We introduce a new independent variable as following

$$
Y=\operatorname{sech}^{2}(\mu \xi), \xi=k_{1} x+k_{2} y-c t,
$$

leads to the change of derivatives in the form

$$
\frac{d}{d \xi}=-2 \mu Y \sqrt{1-Y} \frac{d}{d Y},
$$




$$
\begin{aligned}
\frac{d^{2}}{d \xi^{2}} & =-2 \mu^{2} Y \sqrt{1-Y}\left(\frac{3 Y-2}{\sqrt{1-Y}} \frac{d}{d Y}-2 Y \sqrt{1-Y} \frac{d^{2}}{d Y^{2}}\right) \\
\frac{d^{3}}{d \xi^{3}} & =-2 \mu^{3} Y \sqrt{1-Y}\left(4(1-3 Y) \frac{d}{d Y}+6 Y(2-3 Y) \frac{d^{2}}{d Y^{2}}+4 Y^{2}(1-Y) \frac{d^{3}}{d Y^{3}}\right)
\end{aligned}
$$

where other derivatives can be derived in a similar manner. If we use a new independent variable:

$$
Y=\sec ^{2}(\mu \xi), \xi=k_{1} x+k_{2} y-c t,
$$

leads to the change of derivatives in the form

$$
\begin{aligned}
& \frac{d}{d \xi}=2 \mu Y \sqrt{Y-1} \frac{d}{d Y} \\
& \frac{d^{2}}{d \xi^{2}}=2 \mu^{2} Y \sqrt{Y-1}\left(\frac{3 Y-2}{\sqrt{Y-1}} \frac{d}{d Y}-2 Y \sqrt{Y-1} \frac{d^{2}}{d Y^{2}}\right) \\
& \frac{d^{3}}{d \xi^{3}}=2 \mu^{3} Y \sqrt{Y-1}\left(4(3 Y-1) \frac{d}{d Y}+6 Y(3 Y-2) \frac{d^{2}}{d Y^{2}}+4 Y^{2}(Y-1) \frac{d^{3}}{d Y^{3}}\right) .
\end{aligned}
$$

The $\operatorname{sech}^{2}$ method admits the use of a finite expansion of sech function

$$
u(\mu \xi)=S(Y)=\sum_{k=0}^{m} a_{k} Y^{k},
$$

where $a_{0}, a_{k}(k=1,2, \ldots, m)$ and $\mu$ are constants to be determined later. but, the positive integer $m$ can be determined by considering the homogeneous balance between the highest order derivatives and nonlinear terms appearing in Eq. (10).

Step 3. Substituting Eqs. (12) -(14) or Eqs. (16) -(18) into Eq. (10) with the value of $m$ obtained in Step 2. Collecting the coefficients of $Y^{k}(k=0,1,2, \ldots)$, then setting each coefficient to zero, we can get the set of over-determined nonlinear algebraic equations for $a_{0}, a_{i}(i=1,2, \ldots, n), c$ and $\mu$ with the aid of symbolic computation Maple.

Step 4. Solving the algebraic equations in Step 3, then substituting $a_{0}, \ldots, a_{m}, c$ in Eqs. (15) and (19).

\section{Symmetric Regularized Long Wave (SRLW) Equation}

\subsection{Using the tanh-coth method}

Considering the following Symmetric Regularized Long Wave (SRLW) equation by using the tanh-coth method, we obtain

$$
u_{t t}+u_{x x}+u u_{x t}+u_{x} u_{t}+u_{x x t t}=0
$$


and using the wave variable $\xi=k_{1} x-c t$ reduce it to an ODE

$$
c^{2} u^{\prime \prime}+k_{1}^{2} u^{\prime \prime}-c k_{1} u u^{\prime \prime}-c k_{1}\left(u^{\prime}\right)^{2}+c^{2} k_{1}^{2} u^{\prime \prime \prime \prime}=0 .
$$

Integrating Eq. (21) with respect to $\xi$ and considering the zero constants for integration, we obtain

$$
\left(k_{1}^{2}+c^{2}\right) u^{\prime}-c k_{1} u u^{\prime}+c^{2} k_{1}^{2} u^{\prime \prime \prime}=0 .
$$

Balancing the terms that involve $u^{\prime \prime \prime}$ and $u u^{\prime}$ in Eq. (22) gives

$$
m+3=2 m+1,
$$

so that

$$
m=2 \text {. }
$$

The tanh-coth method allows us to use the substitution

$$
u(\xi)=S(Y)=a_{0}+a_{1} Y+a_{2} Y^{2}+\frac{b_{1}}{Y}+\frac{b_{2}}{Y^{2}} .
$$

Substituting Eq. (25) in to Eq. (22), with the help of Maple gives the following set of non-trivial solutions

$$
a_{0}=\frac{1+c^{2}-8 c^{2} \mu^{2}}{c}, \quad a_{1}=0, \quad a_{2}=12 c \mu^{2}, \quad b_{1}=0, \quad b_{2}=0, \quad k_{1}=1,
$$

or

$$
a_{0}=\frac{1+c^{2}-8 c^{2} \mu^{2}}{c}, \quad a_{1}=0, \quad a_{2}=0, \quad b_{1}=0, \quad b_{2}=12 c \mu^{2}, \quad k_{1}=1,
$$

or

$$
a_{0}=\frac{1+c^{2}-8 c^{2} \mu^{2}}{c}, \quad a_{1}=0, \quad a_{2}=12 c \mu^{2}, \quad b_{1}=0, \quad b_{2}=12 c \mu^{2}, k_{1}=1,
$$

where $c$ and $\mu$ are arbitrary constants. Substituting Eqs. (26)-(28) into expression Eq. (25), can be written as

$$
u_{1}(x, t)=\frac{1+c^{2}-8 c^{2} \mu^{2}}{c}+12 c \mu^{2} \tanh ^{2}[\mu(x-c t)]
$$

or

$$
u_{2}(x, t)=\frac{1+c^{2}-8 c^{2} \mu^{2}}{c}+12 c \mu^{2} \operatorname{coth}^{2}[\mu(x-c t)]
$$

or

$u_{3}(x, t)=\frac{1+c^{2}-8 c^{2} \mu^{2}}{c}+12 c \mu^{2}\left(\tanh ^{2}[\mu(x-c t)]+\operatorname{coth}^{2}[\mu(x-c t)]\right)$. 
It is worth to point out that the following periodic solutions

$$
u_{4}(x, t)=\frac{1+c^{2}+8 c^{2} \mu^{2}}{c}+12 c \mu^{2} \tan ^{2}[\mu(x-c t)],
$$

or

$$
u_{5}(x, t)=\frac{1+c^{2}+8 c^{2} \mu^{2}}{c}+12 c \mu^{2} \cot ^{2}[\mu(x-c t)]
$$

or

$$
u_{6}(x, t)=\frac{1+c^{2}+8 c^{2} \mu^{2}}{c}+12 c \mu^{2}\left(\tan ^{2}[\mu(x-c t)]+\cot ^{2}[\mu(x-c t)]\right) .
$$

which are the exact solutions of symmetric regularized long wave (SRLW) equation. Can be seen that the results are the same, with comparing results Darwish's and Xu's [18].

\subsection{Using the $\operatorname{sech}^{2}$ method}

Considering the following Symmetric Regularized Long Wave (SRLW) equation by using the $\operatorname{sech}^{2}$ method, and proceeding as before we obtain

$$
u_{t t}+u_{x x}+u u_{x t}+u_{x} u_{t}+u_{x x t t}=0
$$

and using the wave variable $\xi=k_{1} x-c t$ reduce it to an ODE

$$
c^{2} u^{\prime \prime}+k_{1}^{2} u^{\prime \prime}-c k_{1} u u^{\prime \prime}-c k_{1}\left(u^{\prime}\right)^{2}+c^{2} k_{1}^{2} u^{\prime \prime \prime \prime}=0 .
$$

Integrating Eq. (36) with respect to $\xi$ and considering the zero constants for integration, we obtain

$$
\left(k_{1}^{2}+c^{2}\right) u^{\prime}-c k_{1} u u^{\prime}+c^{2} k_{1}^{2} u^{\prime \prime \prime}=0,
$$

for simplicity suppose $k_{1}=1$. By a similar derivation as illustrated in the previous section, we obtain

$$
m=2 .
$$

Therefore by use of the $\operatorname{sech}^{2}$ method, we may choose a solution in the form

$$
u(\xi)=S(Y)=a_{0}+a_{1} Y+a_{2} Y^{2}, a_{2} \neq 0 .
$$

Substituting Eq. (39) in to Eq. (37), and by using the well-known software Maple, and equating the coefficients of the powers $Y$, we then get the following algebraic relations:

$-2 c^{2} a_{2}-2 a_{2}+c a_{1}^{2}+12 c^{2} \mu^{2} a_{1}+2 c a_{0} a_{2}-32 c^{2} \mu^{2} a_{2}=0$, $60 c^{2} \mu^{2} a_{2}+3 c a_{1} a_{2}=0$, 
$-a_{1}-4 c^{2} \mu^{2} a_{1}-c^{2} a_{1}+c a_{0} a_{1}=0$.

By using explicitly Eqs. (39) and (40) we solve this system with the aid of the Maple Package, we obtain the system of following results:

$$
a_{0}=\frac{1}{60} \frac{\left(9+36 \mu^{2}\right) a_{2}^{2}+400 \mu^{4}}{a_{2} \mu^{2}}, a_{1}=-3 a_{2}, \quad a_{2}=a_{2}, \quad c=\frac{3}{20} \frac{a_{2}}{\mu^{2}}, \mu=\mu .
$$

Substituting Eq. (41) in Eq. (39) along with (11), we obtain exact travelling wave solution for Eq. (35) of the form:

$$
u(x, t)=\frac{1}{60} \frac{\left(9+36 \mu^{2}\right) a_{2}^{2}+400 \mu^{4}}{a_{2} \mu^{2}}-3 a_{2} \operatorname{sech}^{2} \mu\left(\mathrm{x}-\frac{3}{20} \frac{\mathrm{a}_{2}}{\mu^{2}} \mathrm{t}\right)+a_{2} \operatorname{sech}^{4} \mu\left(\mathrm{x}-\frac{3}{20} \frac{\mathrm{a}_{2}}{\mu^{2}} \mathrm{t}\right) .
$$

If we choose the following solution forms of Eqs. (15)-(18) and insert them into Eq. (37), equating the coefficients of the powers $Y$, then we get the following algebraic relations:

$$
\begin{aligned}
& 2 a_{2}-2 c a_{0} a_{2}-c a_{1}^{2}+2 c^{2} a_{2}-32 c^{2} \mu^{2} a_{2}+12 c^{2} \mu^{2} a_{1}=0, \\
& 60 c^{2} \mu^{2} a_{2}-3 c a_{1} a_{2}=0, \\
& a_{1}+c^{2} a_{1}-4 c^{2} \mu^{2} a_{1}-c a_{0} a_{1}=0 .
\end{aligned}
$$

By the same manipulation as illustrated above, we obtain

$$
a_{0}=-\frac{1}{60} \frac{\left(9-36 \mu^{2}\right) a_{2}^{2}+400 \mu^{4}}{a_{2} \mu^{2}}, a_{1}=-3 a_{2}, a_{2}=a_{2}, \quad c=-\frac{3}{20} \frac{a_{2}}{\mu^{2}}, \mu=\mu .
$$

where $c$ and $\mu$ are arbitrary constants. Substituting Eq. (44) in Eq. (39) along with (15), we obtain exact travelling wave solution for Eq. (35) of the form:

$$
u(x, t)=\frac{1}{60} \frac{\left(9-36 \mu^{2}\right) a_{2}^{2}+400 \mu^{4}}{a_{2} \mu^{2}}-3 a_{2} \sec ^{2} \mu\left(\mathrm{x}+\frac{3}{20} \frac{\mathrm{a}_{2}}{\mu^{2}} \mathrm{t}\right)+a_{2} \sec ^{4} \mu\left(\mathrm{x}+\frac{3}{20} \frac{\mathrm{a}_{2}}{\mu^{2}} \mathrm{t}\right) .
$$

which is the exact solution of symmetric regularized long wave (SRLW) equation. Can be seen that the results are the same, with comparing results Xu's [18].

\section{Conclusion}

In this article we investigated the symmetric regularized long wave (SRLW) equation by the analytical methods. Obtained the solitary wave and periodic wave solutions by the tanh-coth method and the $\operatorname{sech}^{2}$ method. These methods have been successfully applied to obtain some new generalized solitonary solutions to the symmetric regularized long wave (SRLW) equation. The tanh-coth method and the $\operatorname{sech}^{2}$ method are more powerful in searching for exact solutions of NLPDEs. 
Some of these results are in agreement with the results reported specially by Xu's [18]. Also, new results are formally developed in this article. It can be concluded that the these methods are a very powerful and efficient technique in finding exact solutions for wide classes of problems.

\section{Acknowledgement}

The authors would like to thank the referees for their valuable suggestions and comments.

\section{REFERENCES}

1. R. Hirota, The Direct Method in Soliton Theory, Cambridge Univ. Press, 2004.

2. M.J. Ablowitz, P.A. Clarkson, Solitons, nonlinear evolution equations and inverse scattering, Cambridge: Cambridge University Press, 1991.

3. A. M. Wazwaz, Travelling wave solutions for combined and double combined sine-cosineGordon equations by the variable separated ODE method, Appl. Math. Comput, 177 (2006) 755-760.

4. M. Dehghan, J. Manafian, The solution of the variable coefficients fourth-order parabolic partial differential equations by homotopy perturbation method, Z. Naturforsch, 64 (2009) 420-430.

5. M. Dehghan, J. Manafian, A. Saadatmandi, The solution of the linear fractional partial differential equations using the homotopy analysis method, Z. Naturforsch, 65a (2010) 935949.

6. M. Dehghan, J. Manafian, A. Saadatmandi, Solving nonlinear fractional partial differential equations using the homotopy analysis method, Num. Meth. Partial Differential Eq. J, 26 (2010) 448-479.

7. J. H. He, Variational iteration method a kind of non-linear analytical technique: some examples, Int. J. Nonlinear Mech, 34 (1999) 699-708.

8. M. Dehghan, M. Tatari, Identifying an unknown function in a parabolic equation with overspecified data via He's variational iteration method, Chaos Solitons Fractals, 36 (2008) $157-166$

9. M. Dehghan, J. Manafian, A. Saadatmandi, Application of semi-analytic methods for the Fitzhugh-Nagumo equation, which models the transmission of nerve impulses, Math. Meth. Appl. Sci, 33 (2010) 1384-1398.

10. M. Wang, X. Li, J. Zhang, The $\left(\frac{G^{\prime}}{G}\right)$-expansion method and travelling wave solutions of nonlinear evolution equations in mathematical physics, Phys. Lett. A, 372 (2008) 417-423.

11. J. Manafianheris, Exact Solutions of the BBM and mBBM Equations by the Generalized $\left(\frac{G^{\prime}}{G}\right)$-expansion Method Equations, International Journal of Genetic Eng, 2 (2012) 28-32.

12. E. Fan, Extended tanh-function method and its applications to nonlinear equations, Phys. Lett. A, 277 (2000) 212-218.

13. J. Manafian Heris, M. Lakestani, Solitary wave and periodic wave solutions for variants

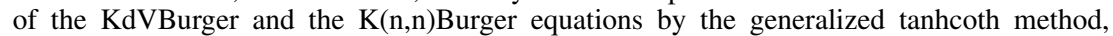
Communications in Numerical Analysis. 2013 (2013) 1-18.

14. X. H. Menga, W. J. Liua, H. W. Zhua, C. Y. Zhang, B. Tian, Multi-soliton solutions and a Bäcklund transformation for a generalized variable-coefficient higher-order nonlinear Schrö dinger equation with symbolic computation, Phys. A, 387 (2008) 97-107.

15. X. Lü, H. W. Zhu, X. H. Meng, Z. C. Yang, B. Tian, Soliton solutions and a Bäcklund transformation for a generalized nonlinear Schrödinger equation with variable coefficients from optical fiber communications, J. Math. Anal. Appl, 336 (2007) 1305-1315. 
16. J. Manafian Heris, M. Bagheri, Exact solutions for the modified KdV and the generalized KdV equations via Exp-function method, J. Math. Extension, 4 (2010) 77-98.

17. J. Manafian Heris, Isa Zamanpour, ANALYTICAL TREATMENT OF THE COUPLED HIGGS EQUATION AND THE MACCARI SYSTEM VIA EXP-FUNCTION METHOD, Acta Universitatis Apulensis, 33 (2013) 203-216.

18. F. Xu, Application of Exp-function method to Symmetric Regularized Long Wave (SRLW) equation, Phsy Comput, 372 (2008) 252-257.

19. M. Dehghan, J. Manafian Heris, A. Saadatmandi, Application of the Exp-function method for solving a partial differential equation arising in biology and population genetics, Int. J. Num. Methods for Heat Fluid Flow, 21 (2011) 736-753.

20. M. Dehghan, J. Manafian Heris, A. Saadatmandi, Analytical treatment of some partial differential equations arising in mathematical physics by using the Exp-function method, Int. J. Modern Phys. B, 25 (2011) 2965-2981.

21. W. Malfliet, Solitary wave solutions of nonlinear wave equations, Am. J. Phys, 60 (1992) 650-654

22. W. Malfliet, Willy Hereman, The tanh method: II. Perturbation technique for conservative systems, Phys. Scr, 54 (1996) 569-575.

23. A.M. Wazwaz, The extended tanh method for new solitons solutions for many forms of the fifth-order KdV equations, Appl. Math. Comput, 184 (2007) 1002-1014.

24. A.M. Wazwaz, The tanh method for generalized forms of nonlinear heat conduction and BurgersFisher equation, Appl. Math. Comput, 169 (2005) 321-338. 